\title{
Ein hinduistischer Tempel unter muslimischer Herrschaft ${ }^{1}$
}

\author{
Von \\ HERMANN KULKE \\ Heidelberg
}

1665 und 1668 fielen die hinduistischen Reiche von Vijayanagara und Orissa im Süden und im Osten Indiens den Angriffen überlegener muslimischer Heere zum Opfer. Damit war die Epoche der mittelalterlichen hinduistischen Regionalreiche in Indien abgeschlossen. In den folgenden anderthalb Jahrhunderten fand dann die islamische Staatenbildung in Südasien im Reich der Moghulen ihren Höhepunkt. Einer der weniger bekannten Aspekte dieser Zeit ist die Rolle, die die sakralen Zentren der früheren hinduistischen Großreiche bei der Entstehung lokaler Nachfolgestaaten spielten, sowie ihre Beziehungen zu den muslischen Provinzgouverneuren. Dieser Aspekt der Geschichte hinduistischer Lokalstaaten im Moghulreich soll hier am Beispiel Orissas und des Jagannātha-Kults in Puri kurz aufgezeigt werden.

Nachdem die afghanischen Heere des Sultāns Sulaimān von Bengalen Zentralorissa erobert und den letzten hinduistischen König von Orissa getötet hatten, überfiel der muslimische General Kalāpāhār mit einem kleinen Heer den berühmten Tempel von Puri². Dort raubte er den Tempelschatz, der nach einem zeitgenössischen Bericht u. a. aus 700 Skulpturen aus purem Gold bestand ${ }^{3}$ und verbrannte das große hölzerne Götterbildnis Jagannāthas. Noch etwa 40 Jahre später pries al-Badā' unī in seiner berühmten Chronik die Afghanen, die „Höhle des Heidentums erobert und das Bollwerk Jagannāthas in eine Stätte des Islams verwandelt zu haben" . Kalāpāhār, der „Schwarze Fels“, gilt in Orissa als Inbegriff des wütenden Ikonoklasten, und es wird noch heute berichtet, daß beim weithin vernehmbaren Ton seiner Kriegstrommeln den Skulpturen der Tempel Orissas Ohren und Nasen abfielen ${ }^{5}$.

Wenn es auch nach einigen Jahrzehnten um 1590 gelang, den Jagannātha-Kult in Puri zu erneuern, so mußten doch die Priester mit ihrem Gott Jagannātha, dem „Herrn der Welt", im 17. und frühen 18. Jh. ein gutes dutzend Male in die unzugänglichen Gebirge Orissas fliehen. Nahezu 30 Jahre war Jagannātha in dieser Zeit von Puri abwesend, bzw. er war zwangsweise in seinem versiegelten Tempel eingeschlossen, wie dies 15 Jahre unter Kaiser Aurangzeb von 1692-1707 geschah.

Es kann deshalb nicht verwundern, daß das Geschick des Jagannātha-Kults geradezu als ein Musterbeispiel für die hindufeindliche Politik muslimischer Herrscher in Indien gilt.

\footnotetext{
1 Erweiterte Fassung eines Vortrages anläßlich des XIX. Deutschen Orientalistentages, Freiburg, 1. 10. 1975. Der Verfasser dankt der Deutschen Forschungsgemeinschaft für ihre großzügige Förderung des Orissa-Projekts im Sonderforschungsbereich 16. Der vorliegende Aufsatz behandelt einen Teilaspekt einer Monographie, deren Manuskript im Juni 1975 abgeschlossen wurde. (JagannāthaKult und Gajapati-Königtum. Ein Beitrag zur Geschichte religiöser Legitimation mittelalterlicher Herrscher in Indien.) - Auflösung der verwendeten Abkürzungen am Schluß der vorliegenden Arbeit.

2 AA, II, S. 140.

${ }^{3}$ Ni'mat Allāh, Makhzzan-i-Afghana, 170 a; zitiert von M. A. Rahim, History of the Afghans in India A. D. 1545-1613 (Karachi 1961) S. $176 \mathrm{f}$.

4 Muntakhhabu-t-Tawärīkh, übersetzt von W. H. Lowe (Calcutta 1924, Bibliotheca Indica, No 97) Vol. II, S. $166 \mathrm{f}$.

5 RS, S. 18.
} 
Eine genaue Analyse der Quellen ergibt jedoch ein wesentlich differenzierteres Bild der Ereignisse in Puri. Denn es zeigt sich statt dessen, daß die Tempelstadt Puri und der Jagannātha-Kult im Kampf der drei islamischen Mächte, die in der zweiten Hälfte des 16. Jh. um die Vormacht in Orissa kämpften - Moghulen, Afghanen und das südindische GolkondaSultanat - eine wichtige Rolle spielte und daß der Moghulkaiser Akbar wesentlich zur Erneuerung des Kultes beigetragen hat. Auch in den folgenden anderthalb Jahrhunderten waren die Kämpfe der moghulischen Provinzgouverneure in Zentralorissa weniger ein Kampf gegen Puri und den Jagannātha-Kult als vielmehr ein Kampf um Puri und um die Kontrolle seines bedeutenden Kultes. Um die folgenden Ausführungen verständlich zu machen, ist es notwendig, einen kurzen Rückblick auf die Geschichte Orissas und des Jagannātha-Kultes vor seiner Vernichtung $1568 \mathrm{zu}$ werfen.

Puri, das noch heute jährlich etwa von einer Million Pilger besucht wird, gehört seit nahezu einem Jahrtausend zu den großen hinduistischen Wallfahrtsstätten Indiens. Anfang des 9. Jh. soll dort bereits Śarikara eines seiner berühmten Klöster gegründet haben. Der Jagannātha-Kult nahm unter Anantavarman Codaganga, der Zentralorissa eroberte und damit die Gañga-Dynastie von Orissa begründete, einen bedeutenden Aufschwung, als Anantavarman um 1135 den heutigen Tempel in Puri errichtete ${ }^{6}$, dessen gewaltiger Tempelturm die gleiche Höhe erreichte wie der bis dahin größte Tempel Indiens, der BṛhadīśvaraTempel in Tanjore in Südindien.

Wenige Jahrzehnte, nachdem 1192 die vereinigten Heere der hinduistischen Reiche Nordindiens von Mohammed von Ghur geschlagen worden waren und darauf ganz Nordindien erstmals unter islamische Herrschaft geriet, tat der Urenkel Coḍagangas, Ananigabhīma III, einen überraschenden Schritt: Er übergab sein Reich dem Gott Jagannātha und regierte unter dessen Oberhoheit (sāmrājya) als dessen irdischer Stellvertreter und Sohn (rāuta, bzw. putra) und erhob damit Jagannātha zur Staatsgottheit des Orissa-Reichs, der nun als der wahre Rāja von Orissa galt. Mehrere Inschriften ${ }^{7}$ und die Mādạ̣ā Pāñji ${ }^{8}$, die berühmte Tempelchronik Puris, berichten übereinstimmend von diesem für die weitere Geschichte Orissas wichtigen Ereignis. Es ist sehr wahrscheinlich, daß die symbolische Übergabe des Reiches an Jagannātha mit der Weihe eines neuen Jagannātha-Tempels um die Jahreswende 1230/31 in Anangabhīmas neuer Hauptstadt Cuttack zusammenhing, die er nur wenige Jahrzehnte nach dem „Fall“ von Benares bezeichnenderweise „Neu-Benares“ (abhinava Vārāṇasī) nanntę

Anaṅgabhïma scheint mit seinem Schritt nicht nur beabsichtigt zu haben, die Legitimation und den Bestand seiner Herrschaft über Orissa zu sichern. Als Stellvertreter des Gottes von Puri erhob er auch einen Führungsanspruch in der hinduistischen Welt. Davon zeugen u. a. zwei bedeutende Stiftungen für einen viṣnuitischen Tempel im fernen südindischen Kanchi-

6 M. M. Chakravarti, The. Date of the Jagannātha Temple in Puri, in: JASB, LXVII, 4 (1898) S. $228 \mathrm{ff}$. und A. Joshi, Jagannāth Temple Inscription of Choḍagangadeva, in: OHRJ, IX, 3/4 (1961) S. 47-50; ferner K. C. Mishra, The Cult of Jagannātha (Calcutta 1971) S. 41.

7 Siehe z. B.: D. C. Sircar, „Bhubaneswar Inscriptions of Bhīmadeva“, No. 1, Zeile 1-3, in: EI, XXX, 6 (1954) S. 232-236.

8 "Als er [Anarigabhīma] in dieser Stadt Cuttack war, übergab Purușottama [= Ananigabhīma] alles dem Gott Jagannātha und blieb dessen Stellvertreter" (e nagara Kațake thāi Śrī Purușottama Śrī Jagannātha Devaniku samasta samarpi rāutapane thāānti), MP, III, S. 27. Siehe auch D. C. Sircar, God Purushottama at Puri and Cuttack, in: J. of Oriental Research, XVII, 4 (1947/48) S. 209-215.

9. C. Sircar, Nagari Plates of Anangabhīma III, Saka 1151 and 1152, Zeile 138, in: EI, XXVIII (1949/950) S. 235-258 und ders., Studies in Religions Life of Ancient and Medieval India, Delhi 1971, S. 62 ff. 
puram $^{10}$, durch die er sich auch außerhalb seines Reiches als Führer und Retter der Hindus bekannt zu machen wünschte, der nun auf Befehl (ādeśa) des „Herrn der Welt" handelt, wie es in einer dieser Inschriften heißt.

1435 usurpierte Kapilendra den Thron in Cuttack und begründete die Sūryavamśa-Dynastie, deren Könige unter dem Namen Gajapatis („Herrn der Elefanten“) bis zum Beginn des 16. Jh. Ostindien vom Ganges bis in die Nähe von Madras beherrschten ${ }^{11}$. Kapilendra ging als mächtigster Hindu-König seiner Zeit in der Entwicklung der Stellvertreter-Ideologie noch einen Schritt weiter als seine Vorgänger der Ganga-Dynastie. Nachdem er die Priester Puris durch reiche Stiftungen und Steuernachlässe für sich gewonnen hatte ${ }^{12}$, ließ er sich von ihnen nun auch als Erwählter Jagannāthas feiern. Zahlreiche Inschriften berichten des weiteren darüber, daß Kapilendra jeweils vor großen Entscheidungen den Gott Jagannātha in Puri um Rat fragte, bzw. sich von den Priestern Puris seine Pläne mit der Autorität des Staatsgottes sanktionieren ließ ${ }^{13}$. Es ist deshalb nur folgerichtig, daß Kapilendra in seinen Inschriften seinen Widersachern mit dem Fluch Jagannāthas drohte und verkünden ließ, daß Widerstand gegen seine königlichen Anordnungen heimtückischer Angriff und Aufruhr (droha) gegen Jagannātha sei. Unter Kapilendra, der damit nicht nur den Staatskult, sondern letztlich auch den Reichsgott als politisches Herrschaftsinstrument einsetzte, fand die Stellvertreter-Ideologie der mittelalterlichen Könige Orissas ihren Höhepunkt. Seither war der oberste hinduistische Rāja in Orissa nicht mehr ohne direkte Beziehungen zu Puri und ohne feste Kontrolle über den Jagannātha-Kult denkbar.

Im frühen 16. Jh. entstanden dann dem Orissa-Reich in dem unter Kṛșnạadeva Rāya erstarkten südindischen Reich von Vijayanagara und den zentral- und nordostindischen Sultanaten von Golkonda und Bengalen fast gleichzeitig drei übermächtige Feinde, deren Angriffe das Reich mehrfach schwer erschütterten. Nachdem dann mehrere blutige Palastrevolten und Verrat höchster Offiziere die Abwehrkraft des Reiches nahezu vollständig gelähmt hatten, gelang es 1568 den Truppen des bengalischen Sultanats, Nord- und Zentralorissa zu erobern, während nahezu gleichzeitig die Heere Golkondas von Süden her bis an die Grenzen des heutigen Orissa bei Chicacole vorstießen und die Provinz Kalinga damit für immer Orissa entrissen.

Die Zerstörung der Götterbildnisse von Puri, der Untergang des Gajapati-Königtums und die Besetzung des Landes durch die Afghanen schien zunächst auch das Ende des Jagannātha-Kultes zu bedeuten, denn zu eng war er kultisch und wirtschaftlich mit dem hinduistischen Königtum in Orissa verbunden gewesen. Doch bereits nach etwa 20 Jahren gelang es einem Rāja Rāmacandra, der in Khurda in der Nähe von Puri nach dem Untergang des Reiches ein kleines Fürstentum begründet hatte, den Kult in Puri wieder feierlich zu erneuern.

Das Datum dieser Kulterneuerung in Puri ist umstritten. Die Historiker Orissas stimmen heute meist darin überein, daß Rāja Rāmacandra diese Tat aus eigener Kraft bereits im Jahr 1575 gelungen sei, nachdem die Afghanen Bengalens im März desselben Jahres erstmals von einem Heer Abkars eine schwere Niederlage hatten hinnehmen müssen ${ }^{14}$. Eine

10 T. V. Mahalingam, Two Eastern Ganga Inscriptions at Kanchipuram, in: EI, XXXI (1955) S. 94-98.

11 P. Mukherjee, The History of the Gajapati Kings of Orissa (Calcutta 1953); R. Subrahmanyam, The Süryavaṃsí Gajapatis of Orissa (Waltair 1957).

12 Zum Beispiel: Puri-Inschrift vom 12. 7. 1459, in: K. P. Tripathi, The Evolution of Oriya Language and Script (Cuttack 1962) S. 265.

${ }_{13}$ Zum Beispiel: Puri-Inschrift vom 25. 4. 1464, ebd. S. 272, wo Kapilendra unbotmäßige Fürsten mit dem Zorn Jagannāthas bedroht.

14 Insbesondere K. N. Mahapatra, Gajapati Ramacandra Deva I, in: OHRJ, VI, 4 (1958) S. 238 f. 
eingehende Analyse aller Quellen - der persischen Chroniken, der Tempelchronik Puris und verwandter Texte ${ }^{15}$ sowie der wenigen Inschriften des späten 16 . Jh. ${ }^{16}$ - ergibt jedoch, daß eine derartige frühe Erneuerung des Kultes durch Rāmacandra auszuschließen ist. Gerade wegen ihrer mehrfachen Niederlagen in Bihar und Bengalen wurde Orissa in den folgenden Jahren für die Afghanen immer mehr ein Rückzugsgebiet, und es besteht kein Anlaß, anzunehmen, daß sie eine Erneuerung des Kultes in Puri geduldet hätten, dessen Vernichtung noch vor wenigen Jahren für sie ein großer Triumph war. Alle Anzeichen sprechen statt dessen für die Richtigkeit der Uberlieferung der Tempelchronik von Puri, die berichtet, daß es Rāmacandra von Khurda erst um 1586 möglich war, die neuen Götterbildnisse zunächst nur in seiner Hauptstadt herstellen zu lassen, da Puri noch fest in den Händen der Afghanen war ${ }^{17}$. Die Erneuerung des Kultes in Puri war erst mehrere Jahre später möglich, nachdem der berühmte Moghul-General Mān Singh nach einem erneuten Sieg über die Afghanen im Sommer 1590 zwar deren Herrschaft über Orissa abermals bestätigte, aber ausdrücklich den Jagannātha-Tempel von Puri und seine Umgebung zum Reichsgut der Moghulen erklärte ${ }^{18}$.

Bisher hat man kaum nach den Ursachen gefragt, die Mān Singh als Gouverneur von Bihar zu dieser seltsamen Waffenstillstandsbedingung veranlaßten, sozusagen am anderen Ende des Herrschaftsbereichs der Afghanen eine hinduistische Tempelstadt zum Reichsgut der Moghulen erklären zu lassen. Auch Abū 'l-Fazl, der diesen Vertrag im Akbarnāma am ausführlichsten schildert, schweigt hierüber. Eine wenig beachtete Inschrift in Südorissa aus dem Jahr 1590 vermag hier vermutlich Aufschluß zu geben ${ }^{19}$. Aus ihr geht hervor, daß ein Heer Golkondas im Frühjahr 1590 das „Niemandsland“ in Südorissa, das bisher zwischen den Herrschaftsgebieten der Afghanen und Golkondas lag, erobert hatte und bis an den Chilka-See südlich von Puri vorgedrungen war, wo es strategisch wichtige Orte einnehmen konnte. Mān Singh muß von diesem Vorstoß Golkondas nach Südorissa gewußt haben, als er im August desselben Jahres überraschend Puri zum Kronland erklären ließ. Offenbar ging es ihm darum, die Tempelstadt Puri den unsicheren Afghanen abzunehmen, um den Verlust dieses auch strategisch wichtigen Ortes an Golkonda zu verhindern, bevor Orissa endgültig dem Moghul-Reich eingegliedert war, was - wie sich schon zwei Jahre später zeigen sollte - das eigentliche Ziel Mān Singhs war.

Nach dieser überraschenden Befreiung Puris von den Afghanen setzte ein Ansturm mehrerer Anwärter auf den Gajapati-Thron von Orissa ein. Rāmacandra von Khurda hatte jedoch einen gewissen Vorsprung, da er bereits vor einigen Jahren die Götterbildnisse der Jagannātha-Trinität in seiner Hauptstadt hatte erneuern lassen. Er erkannte die Gunst der Stunde und ließ bereits wenige Monate später diese Bildnisse im Tempel von Puri weihen und erfüllte damit eine der wichtigsten Voraussetzungen für seine Anerkennung als Nachfolger der Gajapatis von Orissa. Die Tempelchronik von Puri berichtet, daß er um dieses Verdienstes willen von den Priestern Puris nach dem legendären Begründer des Kultes als

15 Cakoḍā Pothi o Cakoḍā Basāṇa ba Cayini Cakoḍā, hrsg. von S. Pattanaik (Cuttack 1959); Jagannātha Kaifiat, Local Records, Vol. 60, S. 303-357, Govt. Oriental Manuscr. Libr. Madras; Account of the Gangavamísa, in: Local Records, Vol. 47.

16 S. N. Rajaguru, Three Inscriptions of the Rulers of the Chalukya Family of Orissa, in: OHRJ, VI, 1 (1957) S. $23 \mathrm{ff}$.; S. C. De, Bilingual Stone Inscription of the Paripada Museum, in: OHRJ, II, 3/4 (1953) S. 94-98.

17 Mit dem Problem der Datierung der Erneuerung des Jagannātha-Kultes in Khurda und Puri setzt sich der Verfasser ausführlich in der in Anm. 1 erwähnten Monographie auseinander.

18 AN, III, S. 880.

10 S. N. Rajaguru (wie Anm. 16). 
„Zweiter Indradyumna" gefeiert wurde und den königlichen Turban aus ihren Händen erhielt ${ }^{20}$.

Die bereits erwähnte Inschrift aus Südorissa berichtet des weiteren, daß im Oktober desselben Jahres 1590 auch ein Vetter des letzten Gajapati im Gefolge des Golkonda-Heeres auftrat und ebenfalls seine Ansprüche auf den Gajapati-Thron anmeldete. Um seine Forderungen zu untermauern, bot er sich in der Inschrift als Verteidiger Puris an und bezeichnete sich sogar, was epigraphisch recht selten belegt ist, als eine göttliche Inkarnation ${ }^{21}$. Die Tempelchronik Puris berichtet ferner, daß ein Sohn des letzten Gajapati sogar nach Delhi gezogen sein soll, um dort seine Ansprüche geltend zu machen.

Die Afghanen hielten ihren Vertrag mit Mān Singh allerdings nur ein Jahr. Bereits im Sommer 1591, nach dem Tod von KKhwāja 'Isā, der den Vertrag mit Mān Singh abgeschlossen hatte, überfielen sie erneut den Jagannātha-Tempel ${ }^{23}$ - zu sehr müssen sie sich offenbar über die inzwischen erfolgte Erneuerung des Kultes erbost haben. Für Mān Singh bot dieser Bruch des Waffenstillstands den Anlaß zu einem groß angelegten Feldzug, dessen Ziel es war, Orissa endgültig in das Moghul-Reich einzugliedern. Als Mān Singh mit seinem Heer bis Cuttack siegreich vorgestoßen war und die Afghanen sich auch in Zentralorissa ergeben hatten, zog er mit einer kleinen Truppe unter dem Vorwand einer Pilgerfahrt nach Puri, um dort Rāmacandra gefangenzunehmen, wiẹ Abū 'l-Fazl im Akbarnāma ausdrücklich berichtet ${ }^{24}$. Doch Rāmacandra hatte den Plan Mān Singhs durchschaut und sich in seine befestigte Hauptstadt Khurda zurückgezogen. Nach einer Weigerung Rāmacandras, persönlich vor Mān Singh zu erscheinen, entsandte der erzürnte Mān Singh sieben seiner Generale mit einem starken Heer gegen Khurda. Nachdem Rāmacandra trotz dieses großen Aufgebotes Khurda mehrere Wochen zu halten vermocht hatte, geschah etwas völlig Unerwartetes: Kaiser Akbar griff zugunsten Rāmacandras in den Kampf ein. Abū 'l-Fazl berichtet hierüber: „On hearing of this [der Belagerung von Khurda] His Majesty - who appreciates dignities - became angry, and issued censures. The Rājah [Mān Singh] recalled his troops, and appologised, Rām Cand, on seeing the graciousness of His Majesty, took the thought of paying his respect ... and visited the Rājah and was treated with respect. " 25

Was mögen nun die Gründe sowohl für Mān Singhs seltsame Pilgerfahrt nach Puri wie für Akbars überraschende Zurechtweisung seines großen Generals zugunsten eines kleinen lokalen Fürsten gewesen sein? Möglicherweise hatte Mān Singh bei der vor zwei Jahren erfolgten Umwandlung Puris zum Moghul-Kronland auch daran gedacht, nach einer zukünftigen Eroberung Gesamtorissas selbst den Kult Jagannāthas in Puri feierlich zu erneuern, um als strenggläubiger Hindu seiner Herrschaft in Ostindien eine besondere Legitimierung zu geben. Immerhin berichtet die Chronik der Dynastie von Mān Singh aus Jaipur in Rajasthan, daß Mān Singh sogar einen Jagannātha-Tempel in Puri errichtet habe ${ }^{26}$. Rāmacandra war Mān Singh aber hierbei ganz offensichtlich zuvorgekommen. $\mathrm{Zu}$ der Enttäuschung hierüber muß noch die Rivalität des hinduistischen Rāja Mān Singh gegenüber

${ }^{20}$ MP, I, S. 63. An anderer Stelle wird Rāmacandra auch „Neuer Indradyumna“ oder gar als dessen Inkarnation (avatāra) gepriesen. (Siehe K. N. Mahapatra (wie Anm. 14) S. 239 und ders., Descriptive Catalogue of the Sanskrit Manuscripts of Orissa [Bhubaneswar 1960] Bd. II, S. CXXVI und 125.) Öber Indradyumna, den legendären Begründer des Jagannātha-Kults, siehe R. Geib, Indradyumna-Legende. Ein Beitrag zur Geschichte des Jagannatha-Kultes (Wiesbaden 1975).

${ }^{21}$ divya-avatāra, S. N. Rajaguru (wie Anm. 16) S. 25, Zeile 25.

22 MP, I, S. 63.

${ }^{23}$ AN, III, S. 934.

24 AN, III, S. 941.

25 AN, III, S. 967.

26 Jaipur Vaṃsāwali, zitiert von R. N. Prasad, Raja Man Singh of Amber (Calcutta 1966) S. 87. Die Angabe ist zweifelsohne falsch, zeigt aber Mān Singhs enges Verhältnis zu Puri. 
Rāmacandra hinzugekommen sein, der nicht nur als Erneuerer des Kultes von Jagannātha, sondern auch als dessen irdischer Vertreter in Orissa gefeiert und anerkannt wurde und damit Mān Singhs Autorität in Orissa schwächte.

Akbar, der sich beim Aufbau seines Reiches in besonders starkem Maß auf Hindu-Fürsten stützte ${ }^{27}$, ging es dagegen bei seinem Eingreifen in das Kampfgeschehen in Orissa offenbar darum, Rāmacandra von Khurda als loyalen und starken Verbündeten zu gewinnen, um durch ihn die südliche Grenze von Orissa gegen das unabhängige Golkonda-Sultanat zusätzlich zu sichern. Akbar war mit den Verhältnissen in Orissa spätestens seit 1565 vertraut, als er Botschafter mit dem letzten Gajapati ausgetauscht hatte, um ihn als Verbuindeten gegen das damalige Sultanat von Bengalen zu gewinnen²8. Akbar wußte daher 1592, welche Rolle der Jagannātha-Kult für die Legitimität des lokalen Hindu-Fürsten in Zentralorissa spielte. Bei der Suche nach einem verbündeten Fürsten in Orissa gegen Golkonda kam deshalb für Akbar nur Rāmacandra von Khurda in Frage, der den Kult in Puri erneuert hatte und für Akbar damit legitimer Nachfolger seines früheren verbündeten Gajapati-Königs von Orissa war. Um die Position Rāmacandras weiter zu stärken, nahm ihn Akbar in den Reichsstand der Manșabdāre auf und erhob ihn damit zum reichsunmittelbaren Fürsten ${ }^{29}$.

Nachdem Mān Singh von Akbar einen Tadel erhalten hatte, ordnete er seine eigenen Pläne jenen seines Kaisers unter und führte sie getreulich aus. In einer umfassenden Neuordnung Orissas umgab Mān Singh das fruchtbare Küstenland (Moghulbandi) mit einem halbkreisförmigen Kranz von halbautonomen Gaḍajāta („burggeborenen“) - Grafschaften. Dreißig dieser kleinen Grafschaften im Süden Orissas wurden Rāmacandra als Lehen übertragen. Khurda, das vor Mān Singh etwa lediglich 1300 Quadratmeilen umfaßt hatte, gebot nun über ein Fürstentum von etwa 14000 Quadratmeilen ${ }^{30}$.

Akbar hatte damit wesentlich zur Sicherung des Jagannātha-Kultes und zur Erneuerung eines Gajapati-Nachfolgestaates in Zentralorissa beigetragen. Grundlagen der Politik Akbars gegenüber Khurda und Puri waren sowohl seine Verständigungspolitik gegenüber den Hindu-Fürsten und seine tolerante Religionspolitik sowie sein Plan, die Grenze zu Golkonda durch ein loyales, lokales Fürstentum zu sichern.

Diese Voraussetzungen für den Aufstieg Khurdas änderten sich jedoch unter Akbars Nachfolgern grundlegend. Neben dem bekannten Wandel, dem die Religionspolitik des Reiches und die Politik gegenüber den Hindufürsten nach Akbars Tod unterworfen waren ${ }^{31}$, sind in Orissa zwei weitere wichtige Punkte zu erwähnen. 1607 wurde Orissa eine eigene Subahdārī-Provinz. Khurda lag damit vom Sitz des Moghul-Subahdārs in Cuttack nur etwa zwei Tagesmärsche entfernt. In Anbetracht des Wandels der kaiserlichen Politik gegenüber den hinduistischen Fürstentümern konnte es daher letztlich nur eine Frage der Zeit sein, bis ein Provinzgouverneur des nahegelegenen Cuttacks versuchen würde, die Gajapatis von Khurda, die sich insgeheim als die eigentlichen Herren Orissas betrachteten, zu unterwerfen und durch Mediatisierung unter ihre direkte Herrschaft zu bringen. Als weiterer Grund für den Wandel der Politik der Moghulen gegenüber Khurda muß das Bestreben Kaiser Jahāngīrs angesehen werden, die Grenzen Orissas nach Süden zu verschieben und jene Fürstenstaaten, die im ehemaligen Südorissa nun unter der Herrschaft Gol-

${ }^{27} \mathrm{H}$. Goetz, The Policy of the Grand Moghuls vis-à-vis the Rajput States, in: Indian Culture, XIV, 3 (1948) S. 91-103.

${ }^{28}$ Muntakhabu-t-Tawārīkh (wie Anm. 4) S. 77 f.

29 AA, S. 548.

so W. W. Hunter, Orissa, or the Vicissitudes of an Indian Province under Native and British Rule (Calcutta 1872) Bd. II, S. 19.

${ }^{31}$ Siehe u. a.: M. L. Roy Choudhury, The State and Religion in Mughal India (Calcutta 1951). 
kondas standen, zu erobern. Hierdurch verlor Khurda seine strategisch wichtige Funktion als Pufferstaat zwischen dem Moghulreich und Golkonda.

Bereits wenige Jahre nach dem Tod Akbars bekamen Khurda und Puri durch mehrere Angriffe diesen Wandel der Reichspolitik drastisch zu spüren. Etwa 1609 überfiel ein Rājputenfürst, der im Dienste des Moghul-Subahdārs in Cuttack stand, während des Wagenfestes $^{32}$ Puri, verschanzte sich im Haupttempel, erbeutete dessen Schatz und zwang den Rāja von Khurda zu demütigenden Friedensbedingungen ${ }^{33}$. Nur zwei Jahre später griff der neue Gouverneur Orissas, ebenfalls ein hinduistischer Rājpute, Khurda an und zwang nach lang anhaltenden Kämpfen den Rāja Puruṣottama von Khurda erneut zur Annahme eines teuer erkauften Friedens. Makaram Khān, der von 1617-1620 Gouverneur Orissas war, führte dann den entscheidenden Schlag gegen die Reichsunmittelbarkeit Khurdas, als er Khurda eroberte und seiner direkten Herrschaft unterstellte ${ }^{34}$. Purusottama von Khurda floh daraufhin in eine Festung in den unzugänglichen Bergen Südorissas, wo er fünf Jahre später verstarb ${ }^{35}$. Die Tempelchronik Puris berichtet, daß während dieser etwa 10 jährigen Kämpfe um Puri und Khurda die Priester von Puri auf Befehl des Rājas von Khurda mehrfach die Götterbildnisse aus ihrem Tempel in Puri entfernten und im Süden Orissas in Sicherheit brachten, wo sie insgesamt über sechs Jahre waren ${ }^{36}$.

Die Kämpłe, die die Provinzgouverneure Kaiser Jahāngīrs in diesen Jahren in Orissa führten, gelten in Orissa als brutale Unterdrückungsmaßnahmen der Moghulen gegen den nationalen Kult der Oriyās und werden häufig mit der Zerstörung des Kultes durch die Afghanen im Jahr 1568 verglichen. Bei genauer Betrachtung zeigt sich aber, daß die Angriffsziele der Kriege unter Jahāngīr der Tempelschatz von Puri und der reichsunmittelbare Status der Khurda-Rājas waren, nicht jedoch eine erneute Vernichtung des JagannāthaKultes. Weder die persischen noch die Oriyā-Chroniken berichten von einer unmittelbaren Gefährdung Puris und des Jagannātha-Kultes während dieser Zeit. Doch wann immer der Khurda-Rāja angegriffen wurde, ordnete er die Entfernung der Götterbildnisse aus Puri an, um seinen wichtigsten Besitz und das Pfand der Legitimation seiner Herrschaft in Sicherheit zu bringen.

Unter Kaiser Shāhjahān kam es in Zentralorissa dann jedoch zu einer spürbaren Beruhigung. Bereits während seines Aufstandes gegen seinen Vater war Shāhjahān 1623 mit einem starken Heer durch Orissa gezogen und hatte den Gouverneur von Orissa vertrieben. Ein Rājputenoffizier aus seinem Gefolge hatte daraufhin zusammen mit dem neuen Rāja von Khurda, Narasimha, die Götterbildnisse wieder im Tempel von Puri feierlich aufstellen lassen ${ }^{37}$. Die folgende 30jährige Herrschaft Shāhjahāns bildete für die Gajapatis von Khurda und den Jagannātha-Kult die friedlichsten Jahre der Moghul-Herrschaft.

Ganz offensichtlich hatte sich in den vergangenen Jahren die Erkenntnis durchgesetzt, daß die langjährige Abwesenheit Jagannāthas von Puri und das damit verbundene Ausbleiben der Pilger letztlich allen schadete, allen voran den Priestern und Pilgerführern von Puri, aber auch dem Rāja von Khurda, dem hierdurch die Kontrolle über Puri zunehmend entglitt und der während seines Kampfes gegen die Mediatisierung ein trauriges Dasein in einem kleinen Fort im bergigen Süden fristen mußte. Wenn wir auch keine Nachricht besitzen, daß die von Akbar abgeschaffte und von Jahāngīr und Shāhjahān teilweise wieder

${ }^{32}$ Das alljährlich im Sommer stattfindende Wagenfest (rathayātrā) ist das bedeutendste religiöse Ereignis von Puri.

${ }^{33}$ Bahāristān-i-Ghaybī by Mīrzā Nathan, übers. von M. J. Borah (Gauhati 1936) Bd. I, S. 35-38.

34 Tūzuk-i-Jahāngīin, übersetzt von A. Rogers, hrsg. von H. Beveridge (Delhi $\left.{ }^{2} 1968\right)$ Bd. I, S. 433.

35 MP, I, S. 66.

36 Ebd.

${ }^{37}$ MP, I, S. 67. 
eingeführte Pilgersteuer bereits in diesen Jahren auch in Puri wieder erhoben wurde, so haben wir doch allen Grund zu der Annahme, daß das Ausbleiben des Pilgerstromes nach Puri auch für die Subahdāre von Cuttack eine schwere finanzielle Einbuße darstellte. Daher kam es in dieser Zeit in der Organisation und Kontrolle des Jagannātha-Kultes in Puri zu einer Verständigung zwischen den Rājas von Khurda und den muslimischen Gouverneuren von Cuttack. So berichtet ein britischer Reisender, der Puri im Jahr 1633 besuchte, daß ein Moghuloffizier während der jährlichen Wagenfeste unter einem Baldachin auf einem der Götterwagen mitfuhr, um offensichtlich den Ablauf des Festes zu kontrollieren ${ }^{\mathbf{3 8}}$.

Rāja Narasimha von Khurda versuchte in den dreißiger Jahren des 17. Jh., den Verlust der Reichsunmittelbarkeit seiner Dynastie und den steigenden Einfluß der Moghuloffiziere in Puri durch die Stärkung seiner eigenen Stellung in Puri und eine Reihe einschneidender Reformen und Neuerungen aufzufangen. Wichtigster Schritt war in dieser Hinsicht die Errichtung eines eigenen Palastes in Puri, womit eine Entwicklung begann, die den Jagannātha-Kult zunehmend zu einem dynastischen Kult der Khurda-Rājas werden ließ. Narasimhas Reformen stießen jedoch auf heftigen Widerstand der Priester in Puri und wohl auch auf das Mißtrauen der Provinzgouverneure in Cuttack, denn 1647 wurde Narasimha von zwei Oriyās und dem stellvertretenden Subahdār in seinem Palast in Puri ermordet. Dabei plünderten die Moghulsoldaten zwar den Palast und den Tempelschatz Jagannāthas - es kam jedoch bezeichnenderweise zu keiner Unterbrechung des Kultes ${ }^{39}$.

Puri und Jagannātha blieben auch verschont, als nach dem Aufstand der Fürsten Orissas während des Erbfolgekrieges am Moghul-Hof 1661 der neue Subahdār Khān-i-Dauran Khurda belagerte und eroberte und den jungen Rāja Mukunda Deva gefangennahm -, von dem Khān-i-Dauran übrigens berichtet, daß er „von allen Zamindaren des Landes wie ein Gott verehrt werde" ${ }^{* 40}$.

An der inzwischen bewährten Ubereinkunft zwischen den Rājas von Khurda, den Priestern Puris und den Gouverneuren der von Delhi so fernen Provinz Orissa vermochte auch Aurangzebs hindufeindliche Religionspolitik nichts grundsätzliches zu ändern. Nach seinem berüchtigten Erlaß aus dem Jahr 1669, alle neuen Hindu-Tempel niederreißen zu lassen und Neubauten zu verhindern, wurden zwar auch in Orissa sogar ältere, kultisch sehr bedeutende Tempel zerstört ${ }^{41}$, doch die Oriyā-Chroniken berichten, daß gerade in dieser Zeit zahlreiche neue Tempel in Puri errichtet wurden. Ein Subahdār soll zwar in diesen Jahren von Cuttack nach Puri aufgebrochen sein, um angeblich den Jagannātha-Tempel zu entweihen. In der Nähe Puris soll er dann jedoch, wie die Tempelchronik Puris berichtet, von einem Blitz zur Umkehr bewegt worden sein. Sein Marsch auf Puri macht ganz den Eindruck, als habe er sich seine weitere inoffizielle Duldung des Kultes durch eine hohe Bestechungssumme abkaufen lassen. Vermutlich erfolgte wegen dieser. Haltung der Offiziere im Jahr 1692 dann ein direkter kaiserlicher Befehl, auch den Jagannātha-Tempel zu zerstören.

Rāja Mukunda Deva von Khurda suchte hierauf jedoch den Provinzgouverneur auf und vereinbarte mit ihm eine „symbolische “ Zerstörung einiger Skulpturen am Eingangstor, die er dann sogar selber beaufsichtigte ${ }^{42}$, willigte ferner in eine offizielle Schließung des Tempels

38 P. Acharya, Brutons Account of Cuttack and Puri, in: OHRJ, X, 3 (1961) S. 46 f.

${ }^{39}$ MP, I, S. 67.

40 Murqat-i-Hassan, S. 184, zitiert von J. N. Sarkar, The History of Orissa in the Seventeenth Century, reconstructed from Persian sources, in: J. Bihar and Orissa Res. Soc., II, 2 (1916) S. 340.

${ }^{41}$ Zum Beispiel Saralā-Tempel in Jhankada und Virajā-Tempel in Jajpur (zerstört 1686 bzw. 1687). Der berühmte Balabhadra-Tempel in Kendrapara wurde bereits $1667 / 68$ zerstört.

42 Tabsirat-ul-Nazirin, zitiert von R. L. Mitra, The Antiquities of Orissa (Calcutta 1875) Bd. II, S. 112. 
ein und lieferte eine sehr wahrscheinlich gefälschte Statue Jagannāthas an den Kaiser Aurangzeb aus. Aus allen Oriyā-Chroniken geht hervor, daß der Jagannātha-Kult dennoch in verkleinerter Form im Tempel fortgeführt wurde, den die Priester durch ein geheimes Tor betraten ${ }^{43}$. Es kann kein Zweifel bestehen, daß dies nur mit Billigung der lokalen. Moghuloffiziere möglich war. Aus diesem Grund rief Aurangzeb daraufhin den Provinzgouverneur aus Cuttack ab und entsandte einen hohen Offizier zur Uberprüfung der Vorgänge nach Puri. Dem Rāja von Khurda gelang jedoch das Meisterstück, selbst diesen Revisor bei seinem Besuch in Puri durch ein Geschenk (bheti) von 30000 Rupien als „Freund zu gewinnen", wie es in einer Oriyā-Chronik heißt ${ }^{44}$. Bis zum Tod Aurangzebs blieb der Tempel zwar weiterhin geschlossen, doch Urkunden belegen, daß in dieser Zeit selbst Fürsten Orissas den Tempel besuchten ${ }^{45}$. Wenige Monate nach Aurangzebs Tod wurde dann im Oktober 1707 der Tempel vom Rāja von Khurda gewaltsam geöffnet und der Kult in seiner vollen Größe wieder aufgenommen ${ }^{46}$. Da dies alles nur in Übereinstimmung mit den lokalen Moghuloffizieren geschehen konnte, zeigen gerade diese Jahre, wie wenig Interesse sie an einer völligen Vernichtung des Kultes selbst in der Zeit des gestrengen Aurangzeb hatten.

Diese stillschweigende Ubereinkunft zwischen den muslimischen Provinzgouverneuren von Orissa und den Rājas von Khurda wurde erst unter den Nawāben von Bengalen aufgegeben, die seit dem Verfall des Moghul-Reiches nach Aurangzebs Tod die Provinz Orissa beherrschten. Vor 1730 eroberten Truppen des Nizāms von Hyderabad Distrikte in Südorissa, die zum Herrschaftsgebiet des Subahdārs von Cuttack gehörten. Taqi Khān, der damals in Cuttack regierte, versuchte nach diesem Gebietsverlust seine Herrschaft wenigstens in Zentralorissa zu festigen, indem er den Khurda-Rāja Rāmacandra II gefangennahm, Teile von dessen Fürstentum seiner direkten Herrschaft unterstellte und damit begann, auch den Kult in Puri stärker zu kontrollieren. Rāmacandra gelang es aber, die Herrschaft über Khurda zurückzuerlangen, nachdem er sich als Stellvertreter des „Herrn der Welt “ während seiner Gefangenschaft zum Islam bekehrt haben soll47. Um die für seine Dynastie geradezu tödliche Ausweitung des Einflusses der Subahdāre auf den Jagannātha-Kult zu verhindern, ließ er, ohne daß irgendeine Gefährdung Puris vorlag, Jagannātha aus dem Tempel in Puri entfernen und suchte mit ihm im Süden Zuflucht. Erst als der Subahdār daraufhin Rāmacandras Sohn als neuen Rāja von Khurda einsetzte, kehrte er 1733 mit Jagannātha zum großen Wagenfest nach Puri zurïck, um dann abermals mit dem „Herrn der Welt" in den Süden zu fliehen. Als er daraufhin zusammen mit Jagannātha etwa drei Jahre im Süden blieb, ernannte der Provinzgouverneur von Cuttack einen Fürsten aus der Nähe Cuttacks zum neuen Gajapati und Rāja von Khurda und ließ von einer kleinen Truppe Jagannātha mit Gewalt wieder zurück nach Puri bringen ${ }^{48}$.

Damit war die eigenartige Situation eingetreten, daß ein muslimischer Provinzgouverneur gegen den Willen des lokalen hinduistischen Rājas einen hinduistischen Kult in einem der berühmtesten Tempel Indiens erneuerte. Der Grund hierfür war, wie wir nun erstmals aus einer Chronik Bengalens direkt erfahren, das Ausbleiben von jährlich 900000 Rupien Pil-

43 Insbesondere Cakoḍā Pothi (wie Anm. 15) S. 20.

44 Ebd.

${ }^{45}$ Jagannātha Sthalavrttāntamu, Govt. Oriental Ms. Libr., Madras, Folios D. No 2612 - R. No 1220 (Überșetzung des Telugu-Textes von S. N. Rajaguru für das Orissa-Projekt, S. 142 und 146). 46 MP, I, S. 71.

47 MP, III, S. 77. Als „Beweis“ für die Bekehrung Rāmacandras gilt heute in Puri ein Bildnis Jagannāthas ("Patita Pāvana“), das am Eingangstor des Tempels angeblich deshalb angebracht wurde, um dem Rāja von Khurda die Verehrung Jagannāthas zu ermöglichen, als ihm als Muslim der Zutritt zum Tempel untersagt war.

48 MP, I, S. 77. 
gersteuern während der Abwesenheit Jagannāthas von Puri ${ }^{49}$. Der Kampf, der unter den Afghanen um 1568 gegen den Jagannātha-Kult begonnen hatte, hatte sich damit eindeutig zu einem Kampf zwischen den Rājas von Khurda und den muslimischen Provinzgouverneuren um Puri und um die Kontrolle seines Jagannātha-Kultes gewandelt.

Wenige Jahre später (1739) gelang es zwar noch einmal einem Angehörigen der KhurdaDynastie nach erheblichen finanziellen Zugeständnissen, vom Provinzgouverneur als Gajapati anerkannt zu werden ${ }^{50}$. Doch die Tage der geschwächten Dynastie waren gezählt, als die Marathen 1751 Orissa eroberten. Während die muslimischen Provinzgouverneure der vorangegangenen anderthalb Jahrhunderte zwangsläufig die Kontrolle zumindest über den Ritus des Jagannātha-Kultes dem lokalen hinduistischen Rāja überlassen mußten - und somit auch dessen "gottgleiche Verehrung" duldeten -, mußte den Marathen als neuen hinduistischen Herren über Orissa diese Stellung der Khurda-Rājas höchst verdächtig erscheinen. Sie nutzten daher die erste Gelegenheit, um die Rājas von Khurda zu entmachten. Als Vīrakeśarī von Khurda 1760 einer Zahlungsverpflichtung nicht nachkommen konnte ${ }^{51}$, übernahmen die Marathen die Verwaltung des Jagannātha-Tempels, besetzten das zu Khurda gehörende Hinterland von Puri und unterstellten die Gaḍajāta-Staaten, die Khurda seit Akbar zu Lehen hatte, ihrer eigenen direkten Herrschaft. Khurda war damit nur wenige Jahre nach Beginn der hinduistischen Herrschaft der Marathen wieder auf den Status einer kleinen Gaḍajāta-Grafschaft zurückgefallen, den es innehatte, bevor Rāmacandra als "Zweiter Indradyumna" den Jagannātha-Kult erneuert hatte und von Akbar zum mächtigsten lokalen Rāja von Zentralorissa erhoben worden war ${ }^{52}$.

\section{Abkürzungsverzeichnis}

$\mathrm{AA}=\overline{\mathrm{A}}$ 'īn-i-Akbarī by Abū 'l-Fazl, Bd. I übers. von H. Blochmann (Calcutta 1873); Bd. II übers. von H. S. Jarret, 2. Aufl. von J. N. Sarkar (Calcutta 1948/49, Bibliotheca Indica).

AN = Akbarnāma by Abū 'l-Fazl, übers. von H. Beveridge, 3. Bd. (Bibliotheca Indica).

$\mathrm{EI}=$ Epigraphia Indica.

JASB = Journal of the Asiatic Society of Bengal.

MP = Mādaḷa Pāñji, hrsg. von A. B. Mohanty (Bhubaneswar 21969).

OHRJ = Orissa Historical Research Journal.

RS = Riyāzu-s-Salātīn by Ghhulam H̦usain Salīm, übers. von M. A. Salam (Calcutta 1902/04, Bibliotheca Indica).

\section{RS, S. $302 \mathrm{f}$.}

${ }^{50} \mathrm{MP}, \mathrm{I}, \mathrm{S} .78$.

${ }^{51} \mathrm{MP}, \mathrm{I}, \mathrm{S} .79$.

${ }^{52}$ Zum Wiederaufstieg der Rājas von Khurda als „Rājas von Puri“ unter britischer Herrschaft im 19. Jh. siehe: $\mathrm{H}$. Kulke, Kings without a Kingdom. The Rajas of Khurda and the Jagannatha Cult, in: South Asia, IV (1974) S. $71 \mathrm{ff}$. 